\title{
Local Accumulation of Axonal Mitochondria in the Optic Nerve Glial Lamina Precedes Myelination
}

\author{
Samantha J. Wilkison 1,2, Cora L. Bright ${ }^{1,2}$, Ricardo Vancini ${ }^{3}$, Daniel J. Song ${ }^{1,2}$, \\ Howard M. Bomze ${ }^{1,2}$ and Romain Cartoni ${ }^{1,2 *}$ \\ ${ }^{1}$ Department of Pharmacology and Cancer Biology, Duke University School of Medicine, Durham, NC, United States, \\ ${ }^{2}$ Department of Ophthalmology, Duke University School of Medicine, Durham, NC, United States, ${ }^{3}$ Department of Pathology, \\ Duke University School of Medicine, Durham, NC, United States
}

OPEN ACCESS

Edited by:

James C. Vickers, University of Tasmania, Australia

Reviewed by: Marcela Votruba, Cardiff University, United Kingdom

Adriana Di Polo,

Université de Montréal, Canada

*Correspondence:

Romain Carton romain.cartoni@duke.edu

Received: 09 March 2021 Accepted: 26 April 2021 Published: 20 May 2021

Citation:

Wilkison SJ, Bright $C L$, Vancini $R$, Song DJ, Bomze HM and Cartoni $R$ (2021) Local Accumulation of Axonal Mitochondria in the Optic Nerve Glial

Lamina Precedes Myelination.

Front. Neuroanat. 15:678501.

doi: 10.3389/fnana.2021.678501
Mitochondria are essential for neurons and must be optimally distributed along their axon to fulfill local functions. A high density of mitochondria has been observed in retinal ganglion cell (RGC) axons of an unmyelinated region of the optic nerve, called the glial lamina (GL) in mouse (lamina cribrosa in human). In glaucoma, the world's leading cause of irreversible blindness, the GL is the epicenter of RGC degeneration and is connected to mitochondrial dysfunction. It is generally accepted that the local accumulation of mitochondria in the GL is established due to the higher energy requirement of unmyelinated axons. Here we revisit the connection between mitochondrial positioning and myelin in RGC axons. We show that the high density of mitochondria in the GL is restricted to larger axons and is established before myelination. Thus, contrary to a longstanding belief in the field, the myelination pattern is not responsible for the establishment of the local accumulation of mitochondria in GL axons. Our findings open new research avenues likely critical to understanding the pathophysiology of glaucoma.

Keywords: retinal ganglion cells, mitochondria, optic nerve, axons, retina

\section{INTRODUCTION}

How neurons allocate resources along the axon is critical for their physiology and survival. Chief among these cellular resources are mitochondria, which must be distributed to specific axonal regions to accomplish local functions. Neurons rely heavily on mitochondria for ATP production (Ames, 2000; Zala et al., 2013), calcium buffering (Rizzuto et al., 2012), and reactive oxygen species management (Angelova and Abramov, 2018). Due to the size of their axonal projection, these important functions must be fulfilled locally in critical areas along the axon (MacAskill and Kittler, 2010; Misgeld and Schwarz, 2017). For example, during development, cortical neurons capture mitochondria at future axonal branch points (Courchet et al., 2013). When axons are growing or re-growing after an injury, mitochondria are re-localized to provide the growth cone with the energy needed (Morris and Hollenbeck, 1993; Misgeld et al., 2007; Zhou et al., 2016). Therefore, it is important to have a clear picture of how mitochondria are distributed in specific axonal regions and to understand what factors regulate this positioning. RGCs are neurons from the central nervous system located in the inner retina that project their axons in the optic nerve to reach their targets in the brain via the optic tract. RGC axons are heterogeneously myelinated: In the proximal region of the optic nerve head called the glial lamina (GL) in mouse and lamina cribrosa in human they are unmyelinated whereas the distal retrolaminar region (RL) of the nerve is fully 
myelinated (Figure 1A). The physiological function of this local lack of myelination is unclear but the importance of this optic nerve region is evidenced by the well-established vulnerability of the GL in glaucoma, the leading cause of irreversible blindness worldwide, in which an early insult to axons in the GL triggers RGC degeneration (Nickells et al., 2012). Of significance, mitochondrial dysfunction has been shown to be important to the pathophysiological mechanism of glaucoma (Chrysostomou et al., 2013; Osborne et al., 2016; Williams et al., 2017). Seminal studies have described a higher abundance of axonal mitochondria in the GL compared to the RL (Bristow et al., 2002; Barron et al., 2004; Yu Wai Man et al., 2005; Yu et al., 2013). Despite the relevance of this axonal region in the context of glaucoma little is known about the mitochondrial landscape in axons of the GL both in adult and during development. Since unmyelinated axons require more ATP to propagate axon potentials (Perge et al., 2009), it has long been accepted that the axonal mitochondrial accumulation in the GL is established in response to the higher energy requirement of unmyelinated axons (Bristow et al., 2002; Barron et al., 2004; Yu Wai Man et al., 2005; Yu et al., 2013). However, direct evidence for this causal relationship is lacking. Here, we sought to investigate this relationship by imaging the positioning of mitochondria in mouse RGC axons throughout different myelination stages. Using two imaging techniques, namely serial block face scanning electron microscopy (SBF-SEM) and light sheet fluorescent microscopy (LSFM), we found that the high abundance of mitochondria in GL axons is observed specifically in larger axons independent of the myelination status. Furthermore, we demonstrated that this mitochondrial accumulation was established early during development between P5 and P6-P8, a stage preceding RGC axon myelination.

\section{MATERIALS AND METHODS}

\section{Animals}

Experiments using mice were approved by the Duke University Institutional Animal Care and Use Committee (protocols A19420-10). The mice were housed under a $12 \mathrm{~h}$ light-dark cycle with ad lib access to food and water. Heat and humidity were maintained within the parameters specified in the National Institute of Health Guide for the Care and Use of Laboratory Animals. Experimental procedures were also consistent with this Guide. STOPf $/$ f-mitoEGFP; Vglut2-Cre (mitoRGC) mice used for LSFM study were generated by crossing STOPf/f mitoEGFP with Vglut2-Cre mice both purchased from The Jackson Laboratory (stock 021429 and 028863, respectively). Wild-type C57BL/6J mice (The Jackson Laboratory stock 000664) were used for the SBF-SEM study.

\section{LSFM Samples Preparation}

The optic nerves underwent immunohistochemistry staining protocol adapted from McKey et al. (2020). Adult mice were transcardially perfused with $1 \mathrm{x}$ PBS then $4 \%$ paraformaldehyde (PFA) and neonatal pups heads were drop fixed for $2 \mathrm{~h}$ in $4 \%$ PFA. Optic nerves were then dissected from the surface of the optic nerve head to the optic chiasm and underwent a sequential dehydration sequence into 100\% methanol. Samples were left in a $66 \%$ Dichloromethane, $33 \%$ methanol mixture overnight. After washing with $100 \%$ methanol, the samples were bleached with 5\% hydrogen peroxide and 95\% methanol then sequentially rehydrated and permeabilized in PTx.2, glycine and DMSO. Nerves were then transferred into blocking solution overnight (PTx.2, 1.5\% donkey serum, 10\% DMSO) and put into primary antibody (PTwH, 5\% DMSO, 3\% donkey serum, Goat antiGFP, Abcam ab5450, 1:1000) for 10 days at 37 degrees Celsius. Following three washes of PTwH, samples were incubated with secondary antibody (PTwH, 3\% donkey serum, Cy3-conjugated Donkey Anti-Goat, Jackson ImmunoResearch Labs 705-165-147, 1:1000) overnight. Samples were then washed three times, with PTwH. Adult optic nerves were dehydrated sequentially (25\%, $50 \%, 75 \%, 100 \% \mathrm{MeOH} / \mathrm{PBS}$, respectively). Once dehydrated, samples are incubated with 66\% DCM and 33\% methanol, washed with 100\% DCM and left in 100\% Dibenzyl ether overnight. Samples were again rehydrated sequentially (100\%, $75 \%, 50 \%, 25 \% \mathrm{MeOH} / \mathrm{PBS}$, respectively) and put into Cubic 1 solution overnight (25\% urea, $20 \%$ quadrol, $15 \%$ Triton X-100, $\mathrm{diH} 2 \mathrm{O}$ ). Samples were then incubated in Cubic 2 solution (25\% urea, $50 \%$ sucrose, $10 \%$ triethanolamine, $\mathrm{diH} 2 \mathrm{O}$ ) over night. Adult and neonatal optic nerves were embedded in 1.8\% agarose using a $1 \mathrm{~mL}$ syringe. Once agarose has solidified, samples were expelled out of the syringe so they can hang in Cubic 2 solution overnight and imaged the next day.

\section{LSFM Acquisition}

Image collection was performed on a Zeiss Lightsheet Z.1equipped with CLARITY 20x objective, $\mathrm{Nd}=1.45$ and $\mathrm{NA}=1.0$, and pco.Edge sCMOS cameras (dual). Illumination was dual sided with 10x NA 0.2 objectives and $561 \mathrm{~nm}$ diode pump solid state laser (set at 5 to $6 \%$ of power) via LBF $405 / 488 / 561 / 638$ quad dichroic and a Zeiss ET600/50 nm emission filters. Cleared optic nerves were mounted from a syringe into an imaging sample holder and submerged in Cubic 2 solution prepared fresh with a average refractive index of 1.45. Depending on the data set, the image pixel size ranged from 0.28 to $0.38 \mu \mathrm{m}$ and Z-stacks were collected with a Z-step ranging from 0.585 to $1.24 \mu \mathrm{m}$. The system was controlled by Zeiss Zen 9.2.0.0 2014 SP1 (black edition) for lightsheet.

\section{LSFM Image Analysis}

The sequence of $z$-stack images was opened in Fiji. The background of each image was then measured using the rectangle tool and the minimum value of the pixel intensity histogram was subtracted from each slice. From the projected $z$-stack image, the pixel intensity along the nerve was measured by drawing a line (line tool) covering the entire nerve.

\section{Serial Block Face Scanning Electron Microscopy (SBF-SEM)}

Wild Type (C57BL/6J) mice were perfused using Ringer Solution for $2 \mathrm{~min}$ (sodium chloride $123 \mathrm{mM}$, calcium chloride $1.5 \mathrm{mM}$, potassium chloride $4.96 \mathrm{mM} \mathrm{pH} 7.3-7.4$ ) followed by a fixative solution $(0.15 \mathrm{M}$ cacodylate, $2.5 \%$ glutaraldehyde, $2 \%$ paraformaldehyde, and $2 \mathrm{mM}$ calcium chloride) for $2 \mathrm{~min}$. After 
A

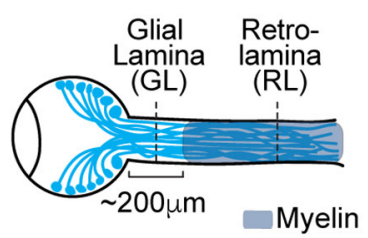

$\mathrm{RL}$

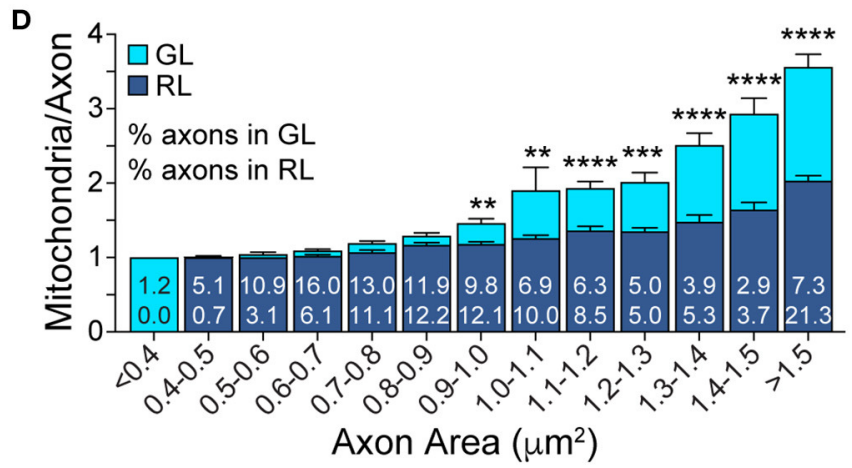

B

GL

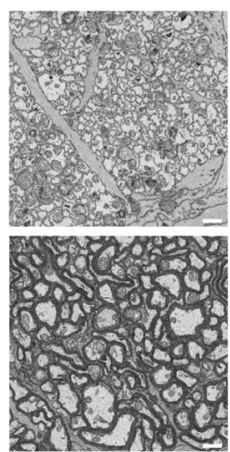

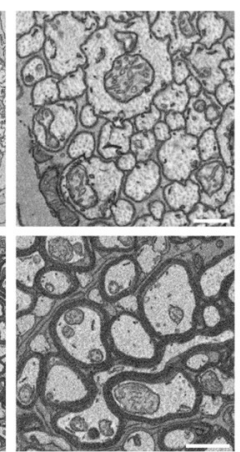

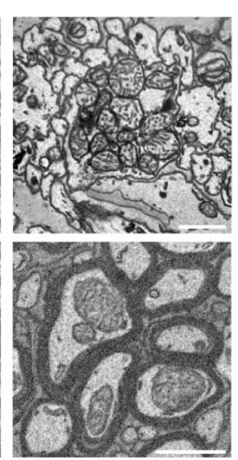

C

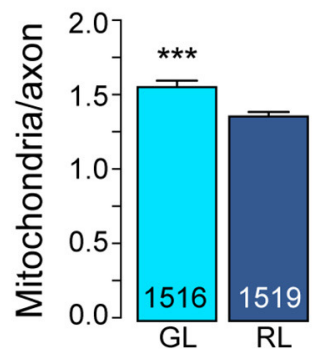

E

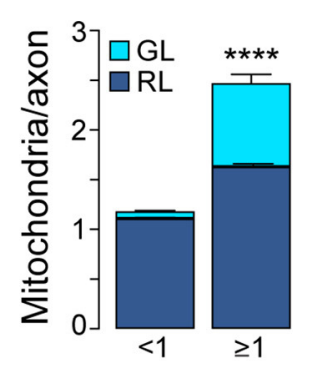

Axon Area $\left(\mu \mathrm{m}^{2}\right)$

FIGURE 1 | The mitochondrial accumulation at the glial lamina is specific to larger axons. (A) Schematic of the adult mouse optic nerve. (B) Representative electron micrographs of optic nerve cross-sections obtained at the unmyelinated $G L$ and myelinated RL regions. Scale bar $=1 \mu \mathrm{m}$. (C) Average number of mitochondria per axon in the GL and RL regions. ${ }^{\star \star \star} p=0.0003$, Mann-Whitney test. The total number of axons are indicated in the bars; $n=3$ mice. (D) GL and RL average number of mitochondria per axon as a function of axonal area. The numbers within each superimposed bar represent the percentage of total axons falling within each area range for the GL (top) and RL (bottom). ${ }^{* *} p<0.002,{ }^{* \star *} p<0.0006,{ }^{* \star \star *} p<0.0001$, Kruskal-Wallis test and Dunn's multiple comparisons. (E) Average number of mitochondria per axon in the GL and RL for axons with areas smaller or bigger than $1 \mu \mathrm{m}^{2}$. ${ }^{\star \star \star \star} p<0.0001$, Kruskal-Wallis test and Dunn's multiple comparisons. Error bars $=$ SEM

fixation, the samples underwent a heavy metal staining protocol adapted from (Deerinck et al., 2010). Briefly, samples were washed in $0.1 \mathrm{M}$ sodium cacodylate $\mathrm{pH} 7.4$ and then transferred for $1 \mathrm{~h}$ at room temperature into a solution of $1.6 \%$ potassium ferrocyanide containing $2 \%$ osmium tetroxide buffered with $0.1 \mathrm{M}$ sodium cacodylate followed by $30 \mathrm{~min}$ in fresh and filtered $10 \%$ thiocarbohydrazide (TCH) solution. Samples were then washed in distilled water and incubated a secondary $2 \%$ aqueous osmium tetroxide incubation for $30 \mathrm{~min}$. The samples were then placed in $1 \%$ aqueous uranyl acetate at $4^{\circ} \mathrm{C}$ overnight, washed in distilled water, and placed in freshly prepared lead aspartate solution $(0.066 \mathrm{~g}$ of lead nitrate in $10 \mathrm{ml} 0.03 \mathrm{M}$ of aspartic acid solution) for $30 \mathrm{~min}$ at $60^{\circ} \mathrm{C}$. The samples were dehydrated in a graded series of cold ethanol, from 25 to $100 \%$ and then infiltrated with increasing concentrations of Durcupan resin in ethanol with several exchanges of $100 \%$ resin. The samples were finally embedded in $100 \%$ resin and allowed to polymerize at $60^{\circ} \mathrm{C}$ for $48 \mathrm{~h}$. The tissue samples embedded in resin were manually trimmed with a razor blade to expose the tissue on their surfaces and then glued onto an aluminum SBF-SEM rivet with conductive epoxy (SPI Conductive Silver Epoxy) with the exposed tissue down. Specimens on the rivet were further trimmed by hand with a razor blade to as small a size as possible (about $0.5 \mathrm{~mm}$ ), and block face was trimmed with a glass knife. Once tissue was exposed, semi thin sections $0.5 \mu \mathrm{m}$ were cut and placed on a glass slide; they were stained with toluidine blue and viewed under a light microscope to check tissue orientation, condition, and correct localization. The rivet with the sample was then sputter coated with gold-palladium to ensure electrical conductivity of the tissue edges with the stub.

\section{Acquisition of SBF-SEM Image Stacks}

The image stacks were acquired in an automated fashion by using a high-resolution field emission scanning electron microscope (SEM) (Merlin-Carl Zeiss, Germany) equipped with a 3View system (Gatan Inc., Pleasanton, CA, USA), and a back-scattered electron detector. Digital Micrograph software (Gatan Inc.) was used to adjust the imaging conditions and slicing parameters. The SEM was operated in the high-resolution mode with an acceleration voltage of $2 \mathrm{kV}$ current mode and in the highvacuum mode. All images were taken at settings of $80 \mathrm{pA}, 2 \mathrm{~s}$ dwell time, and 5-7 nm pixel size. Between 50 and 60 sections were obtained at $60 \mathrm{~nm}$ thickness through $3 \mu \mathrm{m}$ deep, covering fields of view of $75 \times 75 \mu \mathrm{m}$ (neonatal optic nerves) and $100 \times 100 \mu \mathrm{m}$ (adult optic nerve). 


\section{SBF-SEM Image Analysis}

Raw dm4 files were converted to 8bit tiff images and analyzed using Fiji. To assess the axonal size-dependent mitochondrial content, multiple regions of interest were randomly chosen from the original image. The perimeter of each axon analyzed was manually determined using the tracing tool, and the number of mitochondria was counted using the counting tool. Each axon and mitochondrion were given a unique identification number for each image which allowed tracing back to any measurement. For the $\mathrm{G}$ ratio, measurement of myelinated axons, the inner axonal area was divided by the outer total axonal area. For unmyelinated axons, the outer total axonal area was divided by itself.

\section{Statistical Analysis}

Statistical analysis was performed using GraphPad Prism software. Specific sample size, statistical test and $p$-values for each experiment are given in the appropriate figure legends. $P$-value less than 0.05 was considered significant.

\section{RESULTS}

We first used SBF-SEM to image optic nerve cross-sections obtained from the GL and from the RL, respectively, within $150 \mu \mathrm{m}$ and over $300 \mu \mathrm{m}$ from the surface of the optic nerve head (Figure 1A). To have access to a large number of mitochondria spread across a vast population of axons, we imaged a broad field of view of $100 \mu \mathrm{m}$ by $100 \mu \mathrm{m}$. We then quantified the mitochondrial content of axons in the GL and RL in which at least one mitochondrion was identified. Indeed, the percentage of axons with mitochondria was not higher in the GL compared to RL at any age we studied and therefore unlikely to contribute to the high abundance of mitochondria in axons of the GL area (Supplementary Figure 1). In accordance with published studies (Bristow et al., 2002; Barron et al., 2004; Yu Wai Man et al., 2005; Yu et al., 2013), we observed an increase in the mitochondrial content of axons of the GL region compared to RL region (Figures 1B,C). This significant but modest difference led us to hypothesize that the proximal mitochondrial accumulation might not occur uniformly across the axonal population. Although the size distribution of axons differs between the GL and the RL, likely due to the astrocytic web that bundles the axons of the GL, both regions have a wide range of axonal size (from $0.1 \mu \mathrm{m}^{2}$ to over $2 \mu \mathrm{m}^{2}$, data not shown) which likely influences mitochondrial content. We therefore analyzed the number of mitochondria per axon across the range of axonal area and demonstrated that the high mitochondrial content in the GL was restricted to larger axons (Figures 1D,E). Axons with an area above $1 \mu \mathrm{m}^{2}$ show a $51.5 \%$ increase in mitochondrial abundance in the GL (GL: $2.47 \pm 0.09$ vs. RL: $1.63 \pm 0.03$ ) whereas axons smaller than $1 \mu^{2}$ show similar mitochondrial density in the GL and RL (GL: $1.18 \pm 0.01$ vs. RL: $1.11 \pm 0.01$ ) (Figure 1E).

We next tested the paradigm that the absence of myelin around axons in the GL directly induces the local increase in their mitochondrial content. This model predicts that the difference in mitochondrial content between axons in the GL and RL regions should only be apparent after myelination. Therefore, we assessed the mitochondrial density in axons in

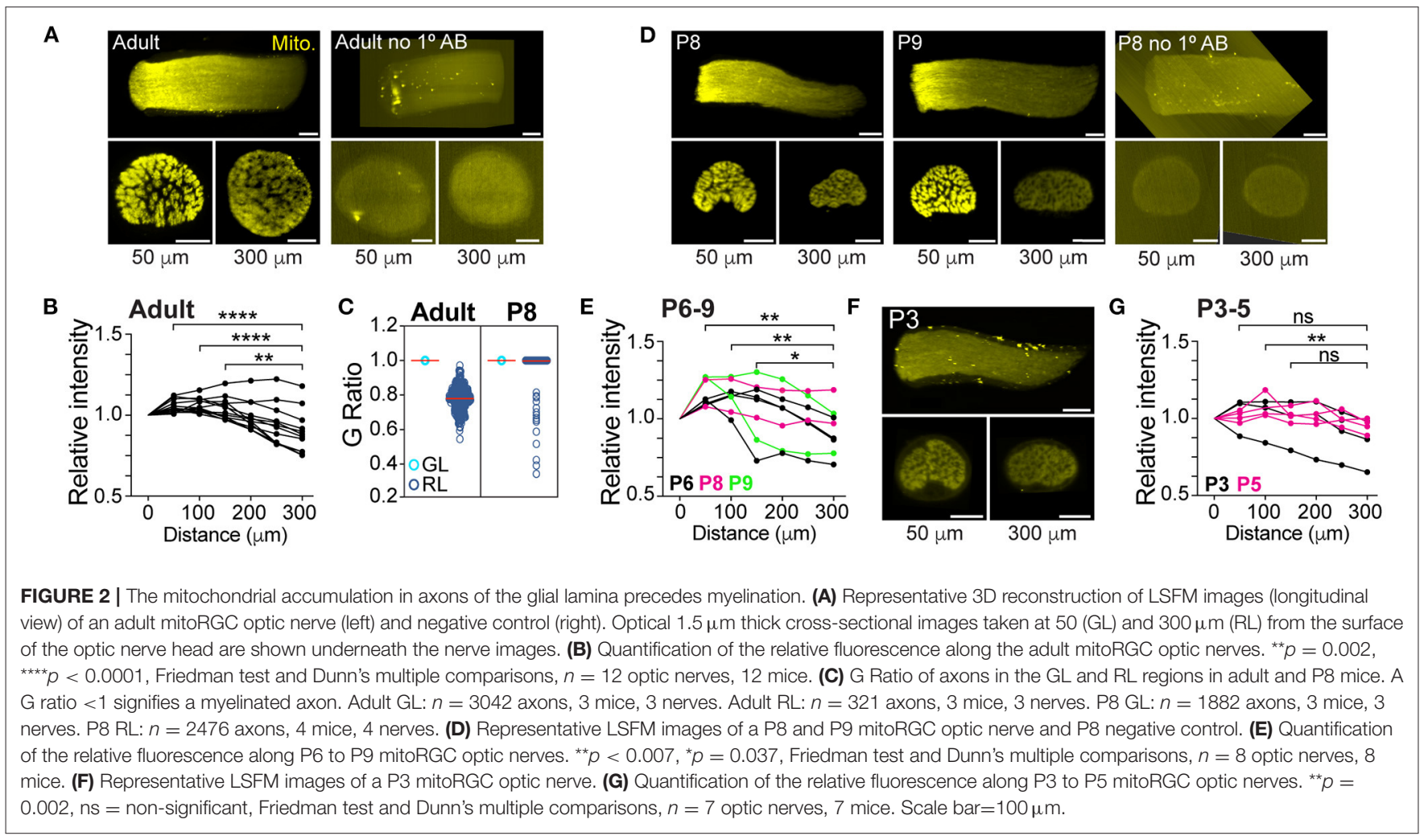


A
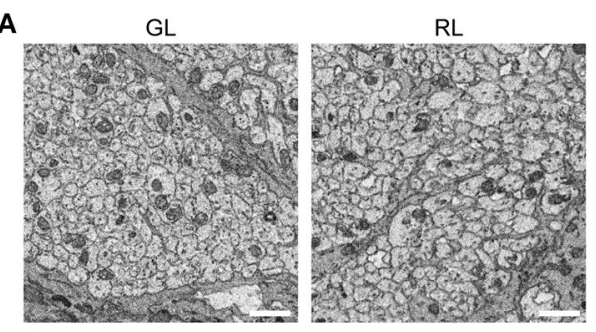

B

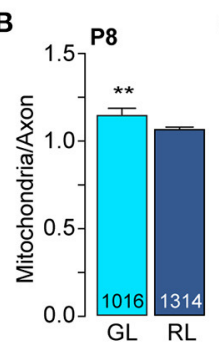

$\mathrm{RL}$
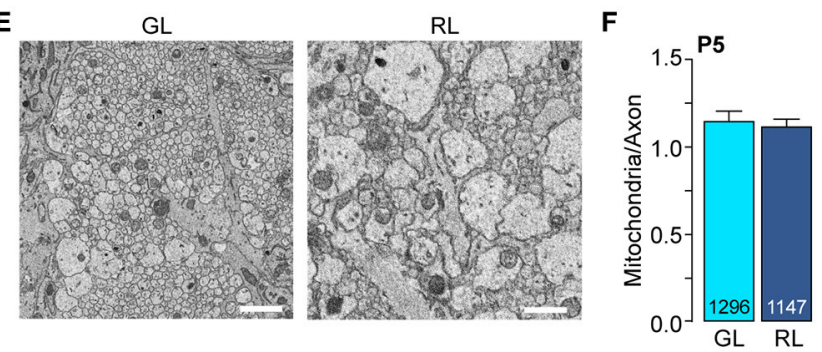

C

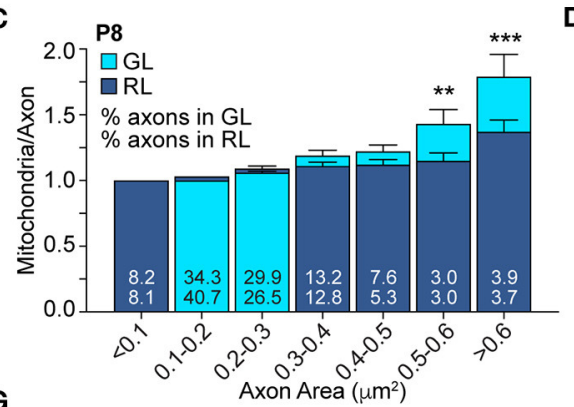

G

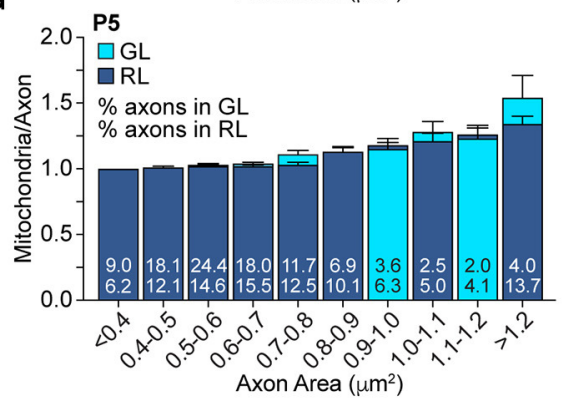

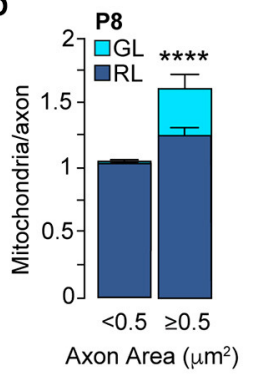

FIGURE 3 | The mitochondrial accumulation in large axons of the glial lamina is established between P5 and P8. (A) Representative electron micrographs of the axons in GL and RL of P8 mice. (B) Average number of mitochondria per axon in axons in GL and RL of P8 mice. The total number of axons is indicated in the bars. GL: $n=3$ mice, 3 nerves. RL: $n=4$ mice, 4 nerves. ${ }^{\star \star \star} p=0.004$, Mann-Whitney test. (C) GL and RL average number of mitochondria per axons in P8 mice split by axonal area. The numbers within each superimposed bar represent the percentage of total axons falling within each area range for the $\mathrm{GL}$ (top) and $\mathrm{RL}$ (bottom). ${ }^{\star \star} p=$ $0.006,{ }^{* \star *} p=0.0002$, Kruskal-Wallis test and Dunn's multiple comparisons. (D) Average number of mitochondria per axon in the GL and RL for axons with areas smaller or bigger than $0.5 \mu \mathrm{m}^{2},{ }^{\star \star \star \star} p<0.0001$, Kruskal-Wallis test and Dunn's multiple comparisons. (E) Representative electron micrographs of the GL and RL axons of P5 mice. (F) Average number of mitochondria per axon in GL and RL of P5 mice. The total number of axons are indicated in the bars. GL: $n=4$ mice, 4 nerves. RL: $n=4$ mice, 4 nerves. (G) GL and RL average number of mitochondria per axon in P5 mice split by axonal area. The numbers within each superimposed bar represent the percentage of total axons falling within each area range for the GL (top) and RL (bottom). Scale bar $=1 \mu \mathrm{m}$. Error bars $=\mathrm{SEM}$.

GL and RL regions of neonatal mice, which have not yet undergone myelination. To efficiently screen multiple neonatal stages, we used LSFM to image the mitochondrial positioning in RGC axons in the intact optic nerves of Vglut2-Cre; STOP $/$ f -mitoEGFP (mitoRGC) transgenic mice that express a mitochondria-targeted EGFP in early embryonic post-mitotic RGCs. As expected, and consistent with our results shown in Figure 1, we observed a mitochondrial enrichment in the first $200 \mu \mathrm{m}$ of the adult mitoRGC optic nerve, corresponding to the GL (Figure 2A left, 2B; Supplementary Video 1). Optical cross sections comparing GL and RL confirmed a non-homogenous axonal distribution of mitochondria between these two regions (Figure 2A, lower panels). MitoRGC optic nerves incubated only with the secondary antibody did not show any signal over background (Figure 2A right, 2D right). Surprisingly, in unmyelinated optic nerves of neonatal mitoRGC mice (P6 to P9) (Figure 2C) we also observed a strong mitochondrial enrichment in proximal axons (Figures 2D,E; Supplementary Video 2). However, this proximal mitochondrial accumulation was absent in P3 to P5 nerves (Figures 2F,G; Supplementary Video 3) in which the level of mitochondrial staining was similar to the RL of adult and P8 (Figures 2A,D).

We next validated this result at single axon resolution using SBF-SEM. We showed that even though $99.7 \%$ of the RL axons are unmyelinated at this age (Figure 2C) we detected an axonal area-dependent increase in mitochondria per axon in the GL of P8 animals (Figures 3A-D), reminiscent of the adult phenotype (Figures 1C-E). Axons larger than $0.5 \mu^{2}$ show a $28.7 \%$ increase in mitochondrial abundance in the GL (GL: $1.64 \pm 0.11$ vs. RL: $1.27 \pm 0.06$ ) whereas it remains constant in axons smaller than $0.5 \mu \mathrm{m}^{2}$ (GL: $1.07 \pm 0.008$ vs. RL: $1.058 \pm 0.007$ ) (Figures 3C,D). Consistent with our results using LSFM, the mitochondrial content was similar in axons of the GL and RL in P5 mice (Figures 3E-G) suggesting that the local accumulation of mitochondria in large GL axons is developmentally regulated and is set between P5 and P6P8, a stage that precedes axon myelination in the optic nerve. Overall, our results demonstrate that from P6-P8 large axons of the optic nerve increase their mitochondria abundance in the GL region comparing to the RL whereas small axons keep a constant number of mitochondria per axons in both regions (Figure 4).

\section{DISCUSSION}

Using two independent approaches, we revisited an old dogma in neuro-ophthalmology regarding the cause of asymmetrical mitochondria distribution in RGC axons. We provided evidence that the preferential accumulation of mitochondria in axons of 


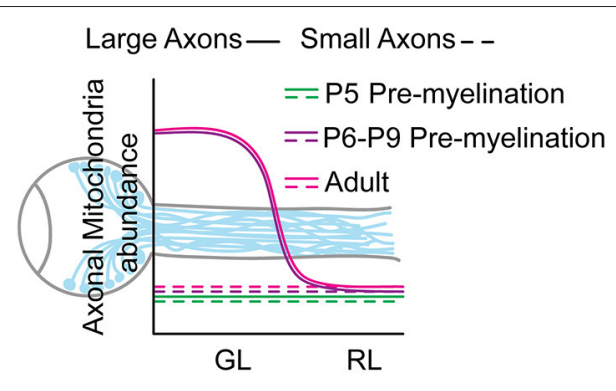

FIGURE 4 | Model of the mitochondrial distribution in RGC axons of the optic nerve $G L$ and $R L$ area.

the GL is restricted to larger axons and is established between P5 and P6-P8. Since this stage precedes RGC axonal myelination of the optic nerve (Mayoral et al., 2018), this result indicates that the establishment of the mitochondrial enrichment in axons in the GL is not a consequence of the local lack of myelination and occurs in absence of the heterogenous myelination pattern of the optic nerve. We cannot rule out that in the adult optic nerve the persistence of high mitochondrial density in large axons in the GL is functionally related to the lack of myelin, since unmyelinated axons require more energy than their myelinated counterpart (Perge et al., 2009). However, our data clearly indicate that a separate mechanism is required for the initial establishment of the asymmetric mitochondrial distribution along the optic nerve. More work is needed to determine any true functional relationship between axonal mitochondrial distribution and myelination pattern. In human optic nerve, the relative distribution of various cytoskeleton proteins appears to be different between the lamina cribrosa and the post laminar region of the optic nerve (corresponding to the GL and RL in mice) (Balaratnasingam et al., 2009). Since mitochondrial positioning largely depends on their interaction with cytoskeleton components such as microtubules and actin filaments (Pathak et al., 2010; Schwarz, 2013), this heterogeneity could play an important role in the mitochondrial enrichment observed in large GL axons. Future studies investigating the local cytoskeletal landscape of GL axons will likely provide important insights into the mechanism by which the local positioning of mitochondria along RGC axons is achieved. If the mitochondrial transport machinery and regulation in neurons is well-characterized (Schwarz, 2013; Misgeld and Schwarz, 2017), much less is known, at least in mammalian cells, about mitochondrial anchoring mechanisms (Kraft and Lackner, 2018). Therefore, it is difficult to speculate on whether the high density of mitochondria in large axons of the GL region is regulated by a specific machinery of mitochondrial proteins such as the axonal specific and canonical mitochondrial anchoring protein syntaphilin (SNPH) (Kang et al., 2008). Interestingly, no expression of SNPH was detected in rat optic nerve axons (Miki et al., 2014) suggesting that RGC axons regulate their stationary pool of mitochondria differently than other neurons of the central nervous system such as cortical neurons (Kang et al., 2008). Proteomic profiling of mitochondria from axons of the GL could help understand how RGC axons anchor their mitochondria in this region. It is also possible that the signaling regulating mitochondrial positioning in axons of the GL is non-cell autonomous. Axons of the optic nerve head use the neighboring astrocytes to degrade dysfunctional mitochondria suggesting a cross talk between RGC axons and astrocytes regarding mitochondria quality control (Davis et al., 2014). Of significance, astrocytes are highly abundant in the GL when compared to the RL. It would therefore be interesting to test whether altering the cellular environment of the GL could affect mitochondrial positioning in GL axons which would indicate a transcellular regulation of mitochondrial positioning in RGC axons. Remarkably, the axonal size specificity of this local accumulation of mitochondria is present in both neonatal and adult optic nerves; within their own axonal range, only large axons in the GL of adult and P8 show a mitochondria abundance higher than in the RL (Figures 1, 3). However, these axons with a mitochondrial accumulation in the GL represent $\sim 7 \%$ of the total axonal population in P8 but $\sim 50 \%$ in adult (Figures 1D, 3C) suggesting that, as the optic nerve grows, the mitochondrial enrichment in larger axons spread across a broader population of axon. It is well-established that unmyelinated axons require more energy than myelinated axons of the same diameter (Perge et al., 2009, 2012). However, our data suggest that smaller axons do not preferentially accumulate mitochondria in unmyelinated regions. This result is consistent with data from adult guinea pig showing that, within the distal optic nerve, the mitochondrial concentration is higher in unmyelinated axons but only in larger axons (Perge et al., 2009). Considerable efforts have been made recently to characterize molecularly the different RGC subtypes (Krieger et al., 2017; Rheaume et al., 2018; Tran et al., 2019) and the function and type specificity of dendritic morphology (Liu and Sanes, 2017; Ran et al., 2020). However, much less is known on the axonal morphology of RGC subtypes, and it is still elusive whether axons of specific size in the optic nerve can be matched to specific RGC subtypes which might reveal common functionality. Therefore, more studies will be needed to decipher if axons accumulating mitochondria proximally in the GL correspond to a combination of RGC subtypes or whether axon size is the only common denominator. Strikingly, the establishment of the local mitochondrial accumulation in large axons of the GL (P6-P8) corresponds to the end of RGC pruning phase, suggesting that the consolidation phase of RGCs and their axons might be coupled with the fine tuning of their mitochondrial landscape (Young, 1984). In glaucoma, RGC death is triggered by an early degeneration of axons in the GL which suggests a local vulnerability likely linked to mitochondria dysfunction (Howell et al., 2007; Williams et al., 2017). How glaucomatous stress affects the local mitochondrial distribution in GL axons is unknown. However, it is tempting to speculate that it might represent an important pathophysiological mechanism. It has been shown that mitochondrial transport in intra-retina RGC axons is impaired in a glaucoma mouse model (Takihara et al., 2015). If the high density of mitochondria in large GL axons is maintained by transporting new mitochondria from the retina, it is therefore possible that under glaucomatous stress 
a transport defect would alter mitochondrial accumulation in GL axons consequently leading to an homogenous distribution of mitochondria along RGC axons which might trigger axonal degeneration in the GL. It will be of major interest to study the physiological relevance of this local mitochondria positioning in a disease context such as glaucoma. Overall, this study brings a new perspective on the regulation of the mitochondrial content in axons of this critical region which is highly relevant to the pathophysiology of glaucoma. It also provides new research avenue on the axonal mechanism of mitochondrial positioning.

\section{DATA AVAILABILITY STATEMENT}

The raw data supporting the conclusions of this article will be made available by the authors, without undue reservation.

\section{ETHICS STATEMENT}

The animal study was reviewed and approved by Duke University Institutional Animal Care and Use Committee (protocols A19420-10).

\section{AUTHOR CONTRIBUTIONS}

SW designed and performed the experiments and analyzed the data. $\mathrm{CB}$ designed and performed experiments and provided assistance in analyzing SBF-SEM data. RV provided technical assistance for tissue preparation and performed the SBF-SEM image acquisition. DS provided assistance on SBF-SEM data analysis and processing. $\mathrm{HB}$ provided technical assistance and intellectual inputs. RC designed the experiments, analyzed the

\section{REFERENCES}

Ames, A. (2000). CNS energy metabolism as related to function. Brain Res. Brain Res. Rev. 34, 42-68. doi: 10.1016/S0165-0173(00)00038-2

Angelova, P. R., and Abramov, A. Y. (2018). Role of mitochondrial ROS in the brain: from physiology to neurodegeneration. FEBS Letters 592, 692-702. doi: 10.1002/1873-3468.12964

Balaratnasingam, C., Morgan, W. H., Johnstone, V., Cringle, S. J., and Yu, D.-Y. (2009). Heterogeneous distribution of axonal cytoskeleton proteins in the human optic nerve. Invest. Ophthalmol. Vis. Sci. 50, 2824-2838. doi: 10.1167/iovs.08-3206

Barron, M. J., Griffiths, P., Turnbull, D. M., Bates, D., and Nichols, P. (2004). The distributions of mitochondria and sodium channels reflect the specific energy requirements and conduction properties of the human optic nerve head. Br. J. Ophthalmol. 88, 286-290. doi: 10.1136/bjo.2003.027664

Bristow, E. A., Griffiths, P. G., Andrews, R. M., Johnson, M. A., and Turnbull, D. M. (2002). The distribution of mitochondrial activity in relation to optic nerve structure. Arch. Ophthalmol. 120, 791-796. doi: 10.1001/archopht.120.6.791

Chrysostomou, V., Rezania, F., Trounce, I. A., and Crowston, J. G. (2013). Oxidative stress and mitochondrial dysfunction in glaucoma. Curr. Opin. Pharmacol. 13, 12-15. doi: 10.1016/j.coph.2012.09.008

Courchet, J., Lewis, T. L. Jr., Lee, S., Courchet, V., Liou, D.-Y., Aizawa, S., et al. (2013). Terminal axon branching is regulated by the LKB1-NUAK1 kinase pathway via presynaptic mitochondrial capture. Cell 153, 1510-1525. doi: 10.1016/j.cell.2013.05.021

Davis, C.-H. O., Kim, K.-Y., Bushong, E. A., Mills, E. A., Boassa, D., Shih, T., et al. (2014). Transcellular degradation of axonal mitochondria. Proc. Natl. Acad. U.S.A. 111, 9633-9638. doi: 10.1073/pnas.1404651111 data, and wrote the manuscript. All authors contributed to the article and approved the submitted version.

\section{FUNDING}

Duke University School of Medicine institutional support (RC), The Whitehead Family Fund (RC), Duke University School of Medicine Core Facility Voucher Program (RC) and the Research to Prevent Blindness Unrestricted Grant (Duke University). National Institute of Health 1S10OD020010-01A1 (Duke Light Microscopy Core Facility), National Institute of Health 1S10OD018439 (Center for Electron Microscopy and Nanoscale Tomography, Department of Pathology, Duke University School of Medicine).

\section{ACKNOWLEDGMENTS}

We thank Dr. McKey for critical help with sample preparation for the LSFM, Dr. Carlson (Duke Light Microscopy Core Facility) for technical assistance with LSFM imaging, Dr. Miller (Center for Electron Microscopy and Nanoscale Tomography, Department of Pathology, Duke University School of Medicine) for assistance with SBF-SEM imaging and samples preparation. Drs. Sidney M. Gospe III, James V. Alvarez, Nahid Iglesias, Jeremy N. Kay and members of the Cartoni lab for comments on the manuscript.

\section{SUPPLEMENTARY MATERIAL}

The Supplementary Material for this article can be found online at: https://www.frontiersin.org/articles/10.3389/fnana. 2021.678501/full\#supplementary-material

Deerinck, T. J., Bushong, E. A., Lev-Ram, V., Shu, X., Tsien, R. Y., Ellisman, M. H. (2010). Enhancing serial block-face scanning electron microscopy to enable high resolution 3-D nanohistology of cells and tissues. Microsc. Microanal. 16, 1138-1139. doi: 10.1017/S1431927610055170

Howell, G. R., Libby, R. T., Jakobs, T. C., Smith, R. S., Phalan, F. C., Barter, J. W., et al. (2007). Axons of retinal ganglion cells are insulted in the optic nerve early in DBA/2J glaucoma. J. Cell Biol. 179, 1523-1537. doi: 10.1083/jcb.200706181

Kang, J.-S., Tian, J.-H., Pan, P.-Y., Zald, P., Li, C., Deng, C., et al. (2008). Docking of axonal mitochondria by syntaphilin controls their mobility and affects short-term facilitation. Cell 132, 137-148. doi: 10.1016/j.cell.2007.11.024

Kraft, L. M., and Lackner, L. L. (2018). Mitochondrial anchors: positioning mitochondria and more. Biochem. Biophys. Res. Commun. 500, 2-8. doi: 10.1016/j.bbrc.2017.06.193

Krieger, B., Qiao, M., Rousso, D. L., Sanes, J. R., and Meister, M. (2017). Four alpha ganglion cell types in mouse retina: function, structure, and molecular signatures. PLoS ONE 12:e0180091. doi: 10.1371/journal.pone.0180091

Liu, J., and Sanes, J. R. (2017). Cellular and molecular analysis of dendritic morphogenesis in a retinal cell type that senses color contrast and ventral motion. J. Neurosci. 37, 12247-12262. doi: 10.1523/JNEUROSCI.2098-17. 2017

MacAskill, A. F., and Kittler, J. T. (2010). Control of mitochondrial transport and localization in neurons. Trends Cell Biol. 20, 102-112. doi: 10.1016/j.tcb.2009.11.002

Mayoral, S. R., Etxeberria, A., Shen, Y.-A. A., and Chan, J. R. (2018). Initiation of CNS myelination in the optic nerve is dependent on axon caliber. Cell Reports 25, 544-550.e3. doi: 10.1016/j.celrep.2018.09.052

McKey, J., Cameron, L. A., Lewis, D., Batchvarov, I. S., and Capel, B. (2020). Combined iDISCO and CUBIC tissue clearing and lightsheet microscopy for 
in toto analysis of the adult mouse ovary ${ }^{\dagger}$. Biol Reprod. 102, 1080-1089. doi: 10.1093/biolre/ioaa012

Miki, A., Kanamori, A., Nakamura, M., Matsumoto, Y., Mizokami, J., and Negi, A. (2014). The expression of syntaphilin is down-regulated in the optic nerve after axonal injury. Experimental Eye Res. 129, 38-47. doi: 10.1016/j.exer.2014.10.017

Misgeld, T., Kerschensteiner, M., Bareyre, F. M., Burgess, R. W., and Lichtman, J. W. (2007). Imaging axonal transport of mitochondria in vivo. Nat. Methods 4, 559-561. doi: 10.1038/nmeth1055

Misgeld, T., and Schwarz, T. L. (2017). Mitostasis in neurons: maintaining mitochondria in an extended cellular architecture. Neuron 96, 651-666. doi: 10.1016/j.neuron.2017.09.055

Morris, R. L., and Hollenbeck, P. J. (1993). The regulation of bidirectional mitochondrial transport is coordinated with axonal outgrowth. J. Cell Sci. 104 (Pt 3), 917-27. doi: 10.1242/jcs.104.3.917

Nickells, R. W., Howell, G. R., Soto, I., and John, S. W. M. (2012). Under pressure: cellular and molecular responses during glaucoma, a common neurodegeneration with axonopathy. Annu. Rev. Neurosci. 35, 153-179. doi: 10.1146/annurev.neuro.051508.135728

Osborne, N. N., Núñez-Álvarez, C., Joglar, B., and del Olmo Aguado, S. (2016). Glaucoma: focus on mitochondria in relation to pathogenesis and neuroprotection. Eur. J. Pharmacol. 787, 127-133. doi: 10.1016/j.ejphar.2016.04.032

Pathak, D., Sepp, K. J., and Hollenbeck, P. J. (2010). Evidence that myosin activity opposes microtubule-based axonal transport of mitochondria. J. Neurosci. 30, 8984-8992. doi: 10.1523/JNEUROSCI.1621-10.2010

Perge, J. A., Koch, K., Miller, R., Sterling, P., and Balasubramanian, V. (2009). How the optic nerve allocates space, energy capacity, and information. J. Neurosci. 29, 7917-7928. doi: 10.1523/JNEUROSCI.5200-08.2009

Perge, J. A., Niven, J. E., Mugnaini, E., Balasubramanian, V., and Sterling, P. (2012). Why do axons differ in caliber? J. Neurosci. 32, 626-638. doi: 10.1523/JNEUROSCI.4254-11.2012

Ran, Y., Huang, Z., Baden, T., Schubert, T., Baayen, H., Berens, P., et al. (2020). Type-specific dendritic integration in mouse retinal ganglion cells. Nat. Commun. 11, 1-15. doi: 10.1038/s41467-020-15867-9

Rheaume, B. A., Jereen, A., Bolisetty, M., Sajid, M. S., Yang, Y., Renna, K., et al. (2018). Single cell transcriptome profiling of retinal ganglion cells identifies cellular subtypes. Nat. Commun. 9:2759. doi: 10.1038/s41467-018-0 $5792-3$

Rizzuto, R., De Stefani, D., Raffaello, A., and Mammucari, C. (2012). Mitochondria as sensors and regulators of calcium signalling. Nat. Rev. Mol. Cell Biol. 13, 566-578. doi: 10.1038/nrm3412
Schwarz, T. L. (2013). Mitochondrial trafficking in neurons. Cold Spring Harb. Perspect. Biol. 5:a011304. doi: 10.1101/cshperspect.a011304

Takihara, Y., Inatani, M., Eto, K., Inoue, T., Kreymerman, A., Miyake, S., et al. (2015). In vivo imaging of axonal transport of mitochondria in the diseased and aged mammalian CNS. Proc. Natl. Acad. Sci. U.S.A. 112, 10515-10520. doi: 10.1073/pnas.1509879112

Tran, N. M., Shekhar, K., Whitney, I. E., Jacobi, A., Benhar, I., Hong, G., et al. (2019). Single-cell profiles of retinal ganglion cells differing in resilience to injury reveal neuroprotective genes. Neuron. 104, 1-17. doi: 10.1016/j.neuron.2019.11.006

Williams, P. A., Harder, J. M., Foxworth, N. E., Cochran, K. E., Philip, V. M., Porciatti, V., et al. (2017). Vitamin B3 modulates mitochondrial vulnerability and prevents glaucoma in aged mice. Science 355, 756-760. doi: $10.1126 /$ science.aal0092

Young, R. W. (1984). Cell death during differentiation of the retina in the mouse. J Comp Neurol. 229, 362-373. doi: 10.1002/cne.902290307

Yu Wai Man, C. Y., Chinnery, P. F., and Griffiths, P. G. (2005). Optic neuropathies-importance of spatial distribution of mitochondria as well as function. Med. Hypotheses. 65, 1038-1042. doi: 10.1016/j.mehy.2004.10.021

Yu, D.-Y., Cringle, S. J., Balaratnasingam, C., Morgan, W. H., Yu, P. K., and Su, E.-N. (2013). Retinal ganglion cells: energetics, compartmentation, axonal transport, cytoskeletons and vulnerability. Progress Retinal Eye Res. 36, 217-246. doi: 10.1016/j.preteyeres.2013.07.001

Zala, D., Hinckelmann, M.-V., Yu, H., da Cunha, M. M. L., Liot, G., Cordelières, F. P., et al. (2013). Vesicular glycolysis provides on-board energyfor fast axonal transport. Cell 152, 479-491. doi: 10.1016/j.cell.2012.12.029

Zhou, B., Yu, P., Lin, M.-Y., Sun, T., Chen, Y., and Sheng, Z.-H. (2016). Facilitation of axon regeneration by enhancing mitochondrial transport and rescuing energy deficits. J. Cell Biol. 104:jcb.201605101. doi: 10.1083/jcb.201605101

Conflict of Interest: The authors declare that the research was conducted in the absence of any commercial or financial relationships that could be construed as a potential conflict of interest.

Copyright (๑) 2021 Wilkison, Bright, Vancini, Song, Bomze and Cartoni. This is an open-access article distributed under the terms of the Creative Commons Attribution License (CC BY). The use, distribution or reproduction in other forums is permitted, provided the original author(s) and the copyright owner(s) are credited and that the original publication in this journal is cited, in accordance with accepted academic practice. No use, distribution or reproduction is permitted which does not comply with these terms. 